\title{
Rapid Detection of Genetically Modified Ingredients in Soybean Products by Real-Time Loop-Mediated Isothermal Amplification
}

\author{
Huiling $\mathrm{Di}^{1}$, Lei Shi ${ }^{1}$, Huiping Shen ${ }^{1}$, He Yan ${ }^{1}$, Hecheng Meng ${ }^{1}$, Lili Li ${ }^{1}$, M. Jahangir Alam ${ }^{2}$, Shinji Yamasaki ${ }^{3}$, \\ Lei Ye ${ }^{1, *}$

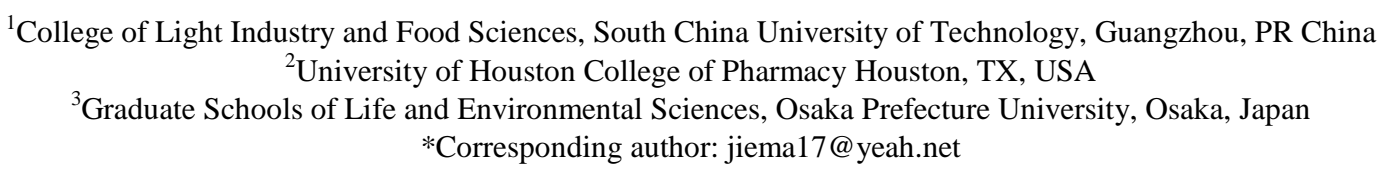

Received June 20, 2014; Revised July 04, 2014; Accepted July 09, 2014

\begin{abstract}
A real-time turbidimeter loop-mediated isothermal amplification (LAMP) assay was developed for the rapid, sensitive, and convenient detection of genetically modified (GM) ingredients in soybean products obtained from local supermarkets in China. Specific primers were designed based on the published sequence of lectin, nos terminator and endogenous genes of two GM soybean strains (GTS40-3-2 and MON89788). The total genomic DNA of soybean-made products was extracted by cetyltrimethylammonium bromide (CTAB) method. Then, the genomic DNA was used as template for LAMP assay. The results showed that this real-time turbidimeter LAMP assay was specific and sensitive enough to amplify DNA from soybean products. The limit of detection (LOD) of lectin and MON89788 was 0.01\% (weight of GM soybean/weight of non-GM soybean), roughly equivalent to four copies of soybean genomic DNA in DNA mixture within one hour; and the LOD of nos and GTS40-3-2 was 0.1\%, roughly equivalent to forty copies of soybean genomic DNA within 1 hour. The sensitivity reaches to $0.01 \%$, which meets the requirements of the European Union (EU). In practical application, the total detectable ratio in soybeanmade products was the same as that of PCR, suggesting that LAMP method was accurate and sensitive.
\end{abstract}

Keywords: real-time turbidimeter-LAMP, GM soybean, rapid detection

Cite This Article: Huiling Di, Lei Shi, Huiping Shen, He Yan, Hecheng Meng, Lili Li, M. Jahangir Alam, Shinji Yamasaki, and Lei Ye, "Rapid Detection of Genetically Modified Ingredients in Soybean Products by Real-Time Loop-Mediated Isothermal Amplification.” Journal of Food and Nutrition Research, vol. 2, no. 7 (2014): 363-368. doi: 10.12691/jfnr-2-7-6.

\section{Introduction}

Agricultural production has grown rapidly in recent years to fulfill the consumer demand of crops. One effective method to ease the burden of the increased demand is to produce genetically modified (GM) crops by introducing novel traits into crops. Production of GM crops has increased dramatically worldwide since 1996, with the cultivate land reaching 1,600,000 $\mathrm{km}^{2}$ in 2011 [1]. According to the International Service for the Acquisition of Agri-biotech Applications (ISAAA), about $10 \%$ of the world's croplands were cultivated with GM crops [2]. In 2011, there were 29 countries involved in the cultivation of GM crops. Of note, $50 \%$ of GM crops were cultivated in developing countries. The United States, Brazil, and Argentina were the three top cultivators of transgenic crops in 2011 [1]. The introduction of GM crops has greatly contributed to the increase in crop yields.

However, with the increase of GM crops worldwide, controversies surrounding GM crops as a public health concern have emerged. GM crops may cause potential hazards to human health and environments, such as incorporation of toxic material or allergens into crops, dissemination of drug resistance, lowering biological diversity and other unknown effects [3]. The increasing public resistance to GM crops has led to the development of strict regulations and accurate detection for transgenic elements in agriculture products. The European Union (EU) has developed clear rules to restrict the proportion of GM ingredients in agriculture products. The proportion of GM ingredients in agriculture products should not exceed $0.9 \%$, and products exceeding this limit must be labeled with GM product to remind consumers [4]. Thus, developing rapid and accurate detection methods for genetically modified organisms (GMOs) in agricultural products were necessary for safe assessments in crop consumption.

The major analysis methods for identification and quantification of GM crops were based on nucleic acid amplification techniques, such as PCR or real-time PCR [5], with good sensitivity and specificity. These methods usually can detect event-specific GMOs such as Mon810, Bt11, or GT73. They also can detect genetic elements such as p35S, tNos, pat, or bar [6-10]. However, these methods usually require expensive equipments and cost 2 or $3 \mathrm{~h}$ to obtain results. 
The recent developed loop-mediated isothermal amplification (LAMP) method is a relatively simple and field-adaptable technique [11]. Target DNA is amplified under isothermal conditions using DNA polymerase with strand displacement property, and Bacillus stearothermophilus (Bst) polymerase is often used. Therefore, sophisticated and expensive thermal cyclers are not required. The amplification of DNA results in the formation of magnesium pyrophosphate which appears as a precipitate as the reaction progresses. The appearance of this precipitate is used as a sign of a positive reaction. In addition, LAMP is shown to amplify DNA with high efficiency, amplifying a few copies of DNA to $10^{9}$ in less than 1 hour [11]. Four specific LAMP primers anneal to six sites of the target sequence [11]. Given that this method does not require a thermo cycler or sophisticated training, LAMP would be an alternative detection assay in both developing and developed countries.

Recently, LAMP was used to detect GMO crops, yielding specific and sensitive results. By targeting eventspecific sequences for transgenic MS8 and RF3 oilseed rape, transgenic element CaMV35S and nopaline synthase terminator (nos), Lee developed a LAMP protocol combined with gel electrophoresis analysis for the detection of GM oilseed rape, with the detection limit reaching 0.01\% [12] (Lee et al. 2009). An optimized visual LAMP method for the detection of two GM soybean events, GTS40-3-2 and MON89788, was reported [13]. Moreover, Liu reported a LAMP method for the detection of 35s epsps in GM soybean, of which the detection limit was 10-fold higher than that of nested PCR [14]. Chen developed a LAMP method to detect specific GMOs, based on the event-specific sequences, in seven GM maize events. The detection limit of this method reached four copies of the maize haploid genomic DNA [15]. Positive LAMP results can be easily observed both by gel electrophoresis or by the naked eye through a color change of the reaction mixture with SYBR green I insertion. However, there are still some disadvantages in result observation by these methods. For instance, it is difficult to determine the LAMP results when the amount of LAMP products is very low. Moreover, the tube cap has to be opened to add SYBR green I to the LAMP products after reaction, which may easily cause aerosol pollution. Hence, it is urgent to develop a simpler, more sensitive, and more accurate LAMP method for the detection of GMOs.

In this study, we developed a real-time turbidimeterLAMP method for rapid and sensitive detection of endogenous soybean gene lectin, nos terminator in two soybean strains, GTS40-3-2 and MON89788, which are in processed soybean products. To our knowledge, this report is the first practical application of the real time LAMP method in process soybean products detection.

\section{Materials and Methods}

\subsection{Materials}

Roundup Ready soybean, GM soybean (GTS40-3-2, MON89788), non-GM soybean, (GM rice, BT63, KF6, GM maize MON89034, BT176, MON810) were provided by Guangdong Entry-exit Inspection and Quarantine Bureau (Guangzhou, China). Non-GM wheat, maize, rice and oilseed rape, bean curd, bean curd skin, soy milk powder, soybean oil, fermented bean curd, fermented soybeans, and soy sauce were purchased from local supermarkets.

\subsection{DNA extraction}

All agricultural food samples were homogenized with a Waring blender. DNA was extracted by cetyltrimethylammonium bromide (CTAB) method [16]. The DNA concentration was measured based on UV absorption at $260 \mathrm{~nm}$, while DNA purity was evaluated on the basis of the UV absorption value ratio at 260/280 nm.

\subsection{Primer design}

All primers were designed by using the specific online software Primer Explorer V3 (http://primerexplorer.jp/ elamp3.0.0/index.html) and synthesized by Invitrogen Co. Ltd. (Shanghai, China). The primers (F3, B3, FIP, and BIP) of each target (lectin, nos for GTS40-3-2 and MON89788) were designed based on the corresponding specific sequences. The primer sequences used in this work were listed in Table 1.

Table 1. LAMP primers used in this study

\begin{tabular}{|c|c|c|}
\hline Gene & primer & Sequence (5' to 3') \\
\hline \multirow[t]{4}{*}{ lectin } & F3 & GCCGAAGCAACCAAACATG \\
\hline & B3 & GGGGCATAGAAGGTGAAGTT \\
\hline & FIP & TGGGGTGCCGTTTTCGTCAACGAGACGCTATTGTGACCTCC \\
\hline & BIP & СТСТАСТССАСССССАTCСACAAAGGAAGCGGCGAAGCT \\
\hline \multirow[t]{4}{*}{ nos } & F3 & CTCGTTCAAACATTTGGCA \\
\hline & B3 & GCGCTATATTTTGTTTTCTATCG \\
\hline & FIP & ACATGCTTAACGTAATTCAACAGAAGATTGAATCCTGTTGCCG \\
\hline & BIP & TGCATGACGTTATTTATGAGATGGCGTATTAAATGTATAATTGCGGGA \\
\hline \multirow{3}{*}{ GTS 40-3-2 } & B3 & GCATTCGAGCTTCTTCAC \\
\hline & FIP & ACAACGAGAAGCTATATGTAGATGCGACCCTAATAGGCAACAGC \\
\hline & BIP & CAAAACTATTTGGGATCGGAGAAGAGAACTTCTCGACGATGGC \\
\hline \multirow[t]{4}{*}{ MON89788 } & F3 & AGCGCTTCAATCGTGGTT \\
\hline & B3 & TGCATGTGCTGGAACAGT \\
\hline & FIP & AGAAGCTTGATAACGCGGCCGCGGGAAACGACAATCTGATCC \\
\hline & BIP & CAGGTCCTGCTCGAGTGGAATGGAGACTCTGTACCCTGA \\
\hline
\end{tabular}

\subsection{LAMP procedure}

The LAMP reaction was carried out in a total reaction volume of $25 \mu$ l containing 50 pmol of each primer (FIP and BIP), 5 pmol of each outer primer (F3 and B3), 1.4 $\mathrm{mM}$ deoxynucleoside triphosphates (dNTPs), $0.8 \mathrm{mM}$ betaine, $0.1 \%$ Tween $20,10 \mathrm{mM}\left(\mathrm{NH}_{4}\right)_{2} \mathrm{SO}_{4}, 8 \mathrm{mM}$ $\mathrm{MgSO}_{4}, 10 \mathrm{mM} \mathrm{KCl,} 20 \mathrm{mM}$ Tris-HCl (pH 8.0, Sigma- 
Aldrich, China), 8 units of Bst DNA polymerase (New England Biolabs., Beijing, China), and $2 \mu \mathrm{l}$ of DNA template. The LAMP reaction was conducted in a a Loopamp Real-time Turbidimeter (LA-320C; Eiken Chemical Co., Ltd., Tokyo, Japan). The mixture was incubating at $63^{\circ} \mathrm{C}$ for $60 \mathrm{~min}$, and then the amplified products were analyzed (Shan et al. 2012).

\subsection{Conventional PCR Procedure}

In conventional PCR, the $25 \mu \mathrm{l}$ of reaction mixture contained PCR buffer, $0.2 \mathrm{mM}$ of each dNTP, $12.5 \mathrm{pmol}$ of each PCR primer, $0.25 \mu \mathrm{l}(5 \mathrm{U} / \mu \mathrm{l})$ of Taq DNA polymerase (TaKaRa Biotech, Dalian, China) and $2 \mu \mathrm{l}$ of DNA template. After denaturation for $5 \mathrm{~min}$ at $94^{\circ} \mathrm{C}$, the PCR mixtures were subjected to 30 cycles of amplification at $94^{\circ} \mathrm{C}$ for $30 \mathrm{~s}, 53^{\circ} \mathrm{C}$ for $45 \mathrm{~s}$ and $72^{\circ} \mathrm{C}$ for $45 \mathrm{~s}$ and a final extension cycle at $72^{\circ} \mathrm{C}$ for $10 \mathrm{~min}$ in a thermal cycler (ABI 2720; Applied Biosystems, Carlsbad, CA, USA). The PCR products were electrophoresed on a $1.5 \%$ agarose gel, and the target bands were visualized by staining with ethidium bromide.

\section{Results}

\subsection{Primer Design and Specificity of LAMP Assay}

One ideal LAMP technique relies on the concurrence of the Bst DNA polymerase with strand displacement activity and a set of four specially designed primers capable of recognizing a total of six distinct regions of the target DNA. As four specific primers existing, the specificity of LAMP is much higher than that of conventional PCR [11]. LAMP primers of GTS 40-3-2 event were designed based on the event-specific sequence of 3' end of exogenous integration (Genebank accession code. AJ308515), recognizing a target sequence of 193 bp [17]. The MON89788 LAMP primers were designed based on event-specific sequence of 3'end of exogenous integration to recognize a target sequence of 259 bp (Genebank accession code. GV597339) [18]. LAMP primers for the soybean endogenous reference gene were targeted at the Lectin sequence (Genebank accession code. K00821), targeting to a target sequence of $207 \mathrm{bp}$. LAMP primers of nos sequence (Genebank accession code. AJ308515), targeting to a target sequence of $203 \mathrm{bp}$. The detailed sequences of all LAMP primers used in this study are listed in Table 1. The optimized conditions of the realtime LAMP assays were described in "method" section. In each LAMP reaction, a total of 100 ng of corresponding plant genomic DNA was used as template.

Lectin is a specific endogenous gene of soybean and only expressed in non-GM soybean or products sourced from non-GM soybean. In the specificity test of lectin detection using LAMP, the typical amplification curve was only obtained from its positive sample (non GM soybean). No curve was observed in other types of plant samples as well as the NTC (Figure 1a). Nos is a marker gene used for the qualitative screening of GM crops. For the specificity test of nos detection using LAMP (Figure 1b), the amplification signal can be observed in the Roundup Ready soybean, GM soybean GTS40-3-2, GM rice BT63, KF6, and GM maize MON89034; while the non-GM soybean, GM soybean MON89788, GM maize BT176 and MON810 did not show any amplification signal. Similarly, in the LAMP assays of GTS40-3-2 and the MON89788, the expected positive results were only obtained from the corresponding positive sample (Figure 1c and 1d). In addition, the LAMP assay showed complete concordance with the conventional PCR method (Figure 1e). It took only $30 \mathrm{~min}$ for the detection of lectin, GTS40-3-2 and MON89788 and 20 to 40 min for nos by using the turbidity real-time LAMP assay. a

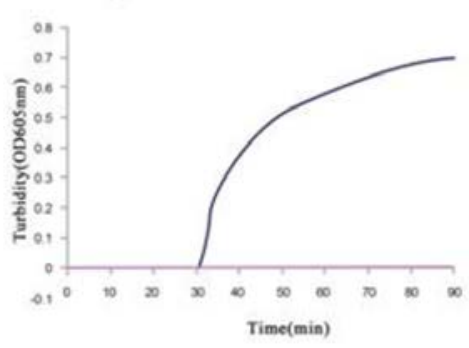

C

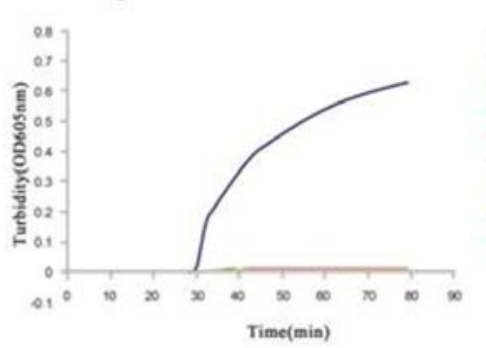

b

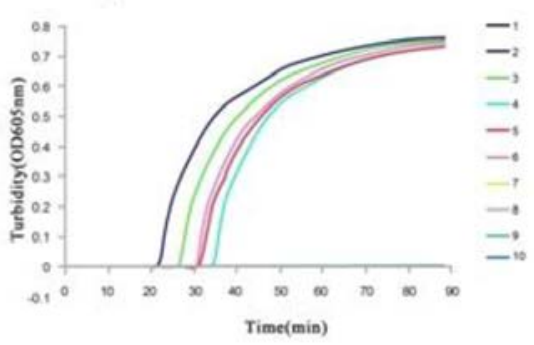

d
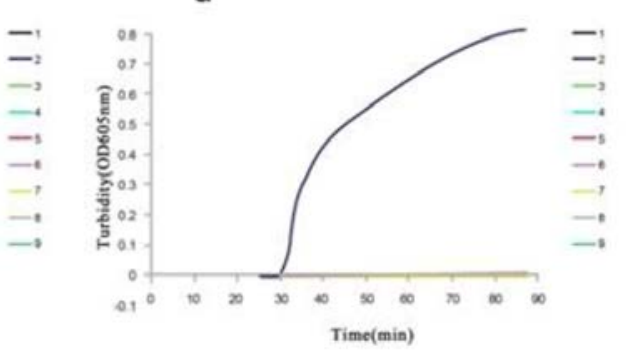

Figure 1. Specificity of real-time turbidimeter LAMP assay. a. Amplification curves of lectin primer set, NO. 1: Positive, NO. 2-6: GM-soybean, wheat, -maize, -rice, oilseed rape; b. Amplification curves of nos primer set, NO. 1: Positive, NO. 2-10: Roundup Ready soybean, GTS40-3-2, BT63, KF6, MON89304, non GM-soybean, MON89788, BT176, MON810; c. Amplification curves of GTS40-3-2 primer set, NO. 1: Positive, NO. 2-9: GTS40-3-2, MON89788, BT63, KF6, MON89304, BT176, MON810, non GM-soybean; d. Amplification curves of MON89788 primer set, NO. 1: negative, NO. 2-9: MON89788, GTS40-3-2, BT63, KF6, MON89304, BT176, MON810, non GM-soybean. e. The electrophoresis gel of lectin gene expression in different samples, Lane 1-6: non-GM soybean, GM soybean, GM wheat, GM maize, GM rice, oilseed rape 


\subsection{Sensitivity}

To determine the limit of detection (LOD) of our assay in the detection of lectin, nos, GTS 40-3-2 and MON89788, the tested samples were prepared by mixing the GM soybean event with non-GM soybean at various ratios, including $100 \%, 10 \%, 5 \%, 1 \%, 0.2 \%, 0.1 \%$ and $0.01 \%$ (weight of $\mathrm{GM} /$ weight of non-GM). In each reaction, a total of $100 \mathrm{ng}$ soybean genomic DNA was

a

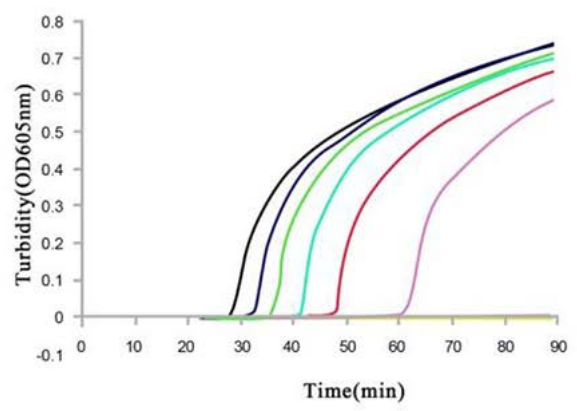

C

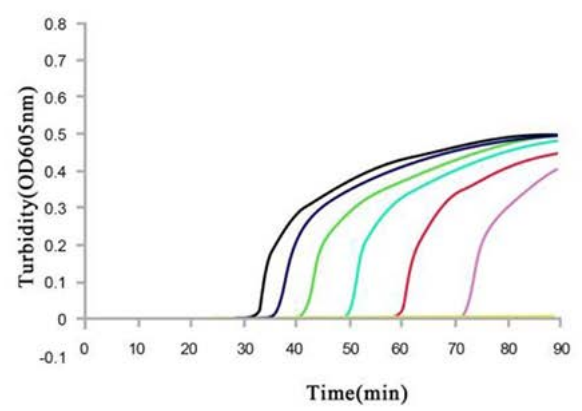

used as amplification template. As shown in Figure 2, the LOD of lectin and MON89788 was $0.01 \%$, roughly equivalent to 4 copies of soybean genomic DNA; and the LOD of nos and GTS40-3-2 was $0.1 \%$, roughly equivalent to 40 copies of soybean genomic DNA. From the amplification curves, the highest optical signals from the four LOD detection appeared in different time, $60 \mathrm{~min}$ for lectin, nos and MON89788 and 75 min for GTS40-3-2.

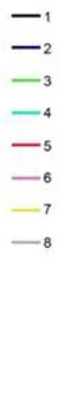

b

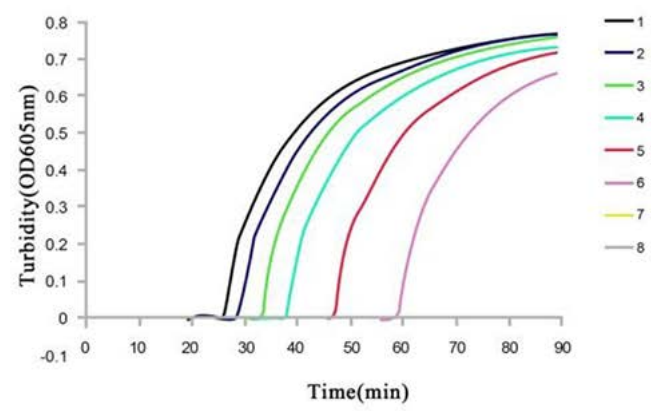

d
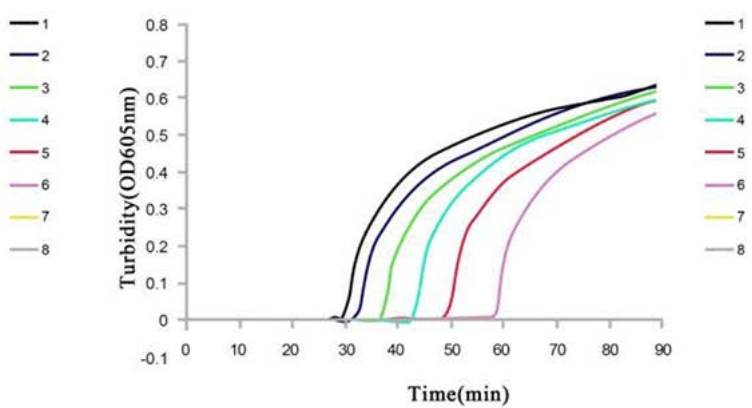

Figure 2. Sensitivity of LAMP assay: a. Amplification curves of lectin primer set, NO.1-7: 100\%, 10\%, 1\%, 0.2\%, 0.1\%, 0.01\% and 0.001\% (the proportion of non-GM soybean), No. 8: blank; b. Amplification curves of nos primer set, NO.1-7: 100\%, 10\%, 5\%, 1\%, 0.2\%, 0.1\% and 0.01\% (the proportion of Roundup Ready soybean), NO. 8: blank; c. Amplification curves of GTS40-3-2 primer set, NO.1-7: 100\%, 10\%, 5\%, 1\%, 0.2\%, 0.1\% and $0.01 \%$ (the proportion of GTS40-3-2 soybean), NO.8: negative; d. Amplification curves of MON89788 primer set, NO.1-7: 100\%, 10\%, 1\%, 0.2\%, $0.1 \%, 0.01 \%$ and $0.001 \%$ (the proportion of MON89788 soybean), NO.8: blank

\subsection{Detection of Transgenic Ingredients in Soybean Products}

Five microliters of the DNA extracted from soybean products were mixed with $495 \mu \mathrm{l}$ distilled water. The values of OD260/OD280 of the genomic DNA from bean curd, bean curd skin, soy milk powder, fermented bean curd and fermented soybeans were between 1.7 and 2.1. It suggested that the extracted genomic DNA from these soybean-products was good enough as amplification template. While the values of OD260/OD280 of the genomic DNA from soy sauce and soybean oil could not be detected. Therefore, we could not detect whether GMOs were included in these two samples.

The results of detection of transgenic ingredients in soybean products were given in Table 2 . For the detection of lectin, 19 out of 22 tested samples were positive for lectin, and three soybean oil samples could not be detected. Three out of 22 tested samples were positive for nos, and the other samples were negative for nos. The LAMP detection of GTS40-3-2 in soybean products showed that 2 out of 22 samples were positive for GTS40-3-2 (two soy milk powders). For the LAMP detection of the MON89788, 2 out of 22 samples were positive for MON89788 (one soy milk powder and one fermented bean curd).

Table 2. LAMP detection results of soybean products

\begin{tabular}{|c|c|c|c|c|c|}
\hline \multirow{2}{*}{ Products } & \multirow{2}{*}{ Number of samples } & \multicolumn{4}{|c|}{ LAMP } \\
\hline & & Lectin $^{+}$ & Nos+ & GTS40-3-2 ${ }^{+}$ & ${\mathrm{MON} 89788^{+}}^{+}$ \\
\hline Bean curd & 3 & 3 & 0 & 0 & 0 \\
\hline Skin of bean curd & 3 & 3 & 0 & 0 & 0 \\
\hline Soy milk powder & 5 & 5 & 2 & 2 & 1 \\
\hline Soy bean oil & 3 & 0 & 0 & 0 & 0 \\
\hline Fermented soy Bean curd & 3 & 3 & 1 & 0 & 1 \\
\hline Fermented soy Bean & 2 & 2 & 0 & 0 & 0 \\
\hline Soy sauce & 3 & 3 & 0 & 0 & 0 \\
\hline Total & 22 & 19 & 3 & 2 & 2 \\
\hline
\end{tabular}


In order to compare the sensitivity of the LAMP assay in soybean products, conventional PCR was performed with the outer primers F3 and B3. In the PCR assay, 16 out of 22 tested samples were positive for lectin, three soybean oil and three soy sauce samples could not be detected by the PCR assay. The PCR results of nos, GTS40-3-2 and MON89788 were consistent with those of LAMP.

\section{Discussion}

Recently, detection of transgenic soybean by the LAMP methods has been reported, and most of these methods needed gel electrophoresis analysis or SYBR Green I for result record. However, the utility of the LAMP assay may be hindered by the low sensitivity of gel or SYBR Green I in the detection of low concentration of target DNA. Besides, both of the methods described above needed to open the tube cap for result analysis which may easily caused aerosol pollution. In order to avoid these disadvantages, we developed a real-time turbidimeter LAMP assay for rapid screening of genetically modified ingredients in processed soybean products in China. Results suggested that our method could amplify DNA with high specificity and sensitivity under isothermal conditions, and the result record was done just at the end of amplification reaction without need of other steps. The LAMP method we developed was much quicker, only 2060 mins, as compared with 90-120 mins for PCR. According to the EU regulations, crops or food that contains more than $0.9 \%$ GMOs products should be labeled with GM to remind consumers [3], and the LOD of our method was equal to or less than $0.1 \%$, which meets the EU requirements. The assay sensitivity in our study was sensitive enough for detection of GMOs in real applications.

The LAMP reaction can be accelerated, and higher sensitivity would be expected by using additional primer, termed loop primer. We tried to added loop primers to a LAMP reaction at first. However, the incidence of false positive was increased. Moreover, the complete LAMP detection system presented here was faster than conventional PCR method. Although the reaction time of LAMP assay with loop primers is reduced, it does not significantly reduce the total assay time. In order to ensure the accuracy of the assay, we have to omit the loop primers.

For practical detection, all of the processed soybean products we chosen in this study are popular traditional food in China, which is filled with complicated components such as proteins, polysaccharides and lipids. These components may inhibit LAMP reaction, therefore, DNA extraction before LAMP reaction is necessary and should be well enough to eliminate such impurity from food samples. Suitable DNA extraction methods in GM crop analysis must be capable of isolating DNA with high yield and purity in order to detect even traces of GM components in subsequent amplification. Cetyltrimethylammonium bromide (CTAB) were proofed to be suitable for an optimal chemical pulping of plant cells, and commonly used for DNA extraction from plant material $[19,20]$. In our study, CTAB was chosen to extract DNA from soybean products, and the results suggested that the purity of DNA extracted by CTAB method can satisfy the needs of the LAMP assay. The possible reason for the failure to detect OD values from soy sauce and soybean oil samples might be the contamination of coloring components in DNA samples. Besides, DNA may have also been destroyed by hightemperature, high-pressure and other special processing. The results indicated that further optimization of the DNA extraction methods that suitable for highly processed food samples were still required in order to extract trace amounts of DNA in processed food.

The GTS40-3-2 and MON89788-positive results of the three soybean products indicated that the soybean products from local supermarkets in China contain transgenic elements. China is in the process of labeling imported GM crops (soybean and oilseed rape) [21], and several GM soybean events such as RRS, MON89788 and GTS40-3-2 have been approved in China. The number of approved GM soybean events is expected to steadily increase, and specific analytical methods for novel GM events or a screening method for GM soybean events will be required. Here, we successfully established a rapid and efficient assay for the detection of GM elements in processed soybean products using the LAMP assay.

\section{Acknowledgements}

This work was supported by the Major State Basic Research Development Program of China (973 Program) (no. 2010CB35704; 2012CB723705), Guangzhou technological planning projects (11C12080718), the Fundamental Research Funds for the Central Universities, SCUT (2012ZZ0083), and the Food Safety Key Lab of Liaoning Province Open Project Funding (LNSAKF2011002).

\section{References}

[1] James, C. "Global Status of Commercialized Biotech/GM Crops: 2011. ISAAA Brief 43-2011. [Online] Available: http://www.isaaa.org/resources/publications/briefs/43/executivesu mmary/default.asp.

[2] James, C. "Global status of commercialized biotech/ GM crops: 2010. ISAAA Brief 43-2011. [Online] Available: http://www.isaaa.org/kc/cropbiotechupdate/specialedition/2011/de fault.asp.

[3] Bakshi, A, "Potential adverse health effects of genetically modified crops," Journal of Toxicology and Environmental Health Part B, Critical Reviews, 6 (3). 211-226. Jan. 2003.

[4] EC. "Regulation (EC) No 1829/2003 of the European Parliament and of the Council of 22 September 2003 on genetically modified food and feed," Official Journal of the European Union, 46. L268. Sep. 2003.

[5] EC. "Commission recommendation 2004/787/EC of 4 October 2004 on technical guidance for sampling and detection of genetically modified organisms and material produced from genetically modified organisms as or in products in the context of Regulation (EC) No. 1830/2003," Official Journal of the European Union, 18-26. Oct. 2004.

[6] Gachet, E., Martin, G., Vigneau, F., Meyer, G., "Detection of genetically modified organisms (GMOs) by PCR: a brief review of methodologies available," Trends in Food Science \& Technology, 9. 380-388. Jan. 1998.

[7] Permingeat, H.R., Reggiardo, M.I., Vallejos, R.H., "Detection and quantification of transgenes in grains by multiplex and real-time PCR,” Journal of Agricultural and Food Chemistry, 50 (16). 4431-4436. Aug. 2002. 
[8] James, D., Schmidt, A.M. Wall, E, Green, M. Masri, S. "Reliable detection and identification of genetically modified maize, soybean, and canola by multiplex PCR analysis,” Journal of Agricultural and Food Chemistry, 51 (20). 5829-5834. Aug. 2003.

[9] Mannelli, I., Minunni, M., Tombelli, S., Mascini, M., "Quartz crystal microbalance (QCM) affinity biosensor for genetically modified organisms (GMOs) detection,” Biosensors and Bioelectronics, 18 (2-3). 129-140. Jun. 2003.

[10] Taverniers, I., Windels, P., Vaitilingom, M., Milcamps, A., Van Bockstaele, E., Vanden Eede, G., De Loose, M., "Event-specific plasmid standards and real-time PCR methods for transgenic Bt11, Bt176, and GA21 maize and transgenic GT73 canola,” Journal of Agricultural and Food Chemistry, 53 (8). 3041-3052. May. 2005.

[11] Notomi, T., Okayama, H., Masubuchi, H., Yonekawa, T., Watanabe, K., Amino, N., Hase, T., "Loop-mediated isothermal amplification of DNA,” Nucleic Acids Research, 28 (12). e63-e63. Jun. 2000.

[12] Lee, D., La Mura, M., Allnutt, T.R., Powell, W., "Detection of genetically modified organisms (GMOs) using isothermal amplification of target DNA sequences,” BMC Biotechnology, 9. 7. Feb. 2009.

[13] Guan, X., Guo, J., Shen, P., Yang, L., Zhang, D., "Visual and rapid detection of two genetically modified soybean events using loop-mediated isothermal amplification method," Food Analytical Methods, 3 (4). 313-320. Jan. 2010.

[14] Liu, J., Guo, J., Zhang, H., Li, N., Yang, L., Zhang, D. "Development and in-house validation of the event-specific polymerase chain reaction detection methods for genetically modified soybean MON89788 based on the cloned integration flanking sequence,” Journal of Agricultural and Food Chemistry, 57 (22). 10524-10530. Oct. 2009.

[15] Chen, L., Guo, J., Wang, Q., Kai, G., Yang, L., "Development of the visual loop-mediated isothermal amplification assays for seven genetically modified maize events and their application in practical samples analysis,” Journal of Agricultural and Food Chemistry, 59 (11). 5914-5918. Jun. 2011.

[16] Doyle, J.J., Doyle, J.L., "A rapid DNA isolation procedure for small quantities of fresh leaf tissue," Phytochemical Bulletin, 19 (1). 11-15. 1987.

[17] Windels, P., Taverniers, I., Depicker, A., Van Bockstaele, E., De Loose, M., "Characterisation of the Roundup Ready soybean insert, ” European Food Research and Technology, 213 (2). 107-112. Aug. 2001.

[18] Liu, M., Luo, Y., Tao, R., He, R., Jiang, K., Wang, B., Wang, L. "Sensitive and rapid detection of genetic modified soybean (Roundup Ready) by loop-mediated isothermal amplification," Bioscience, Biotechnology, and Biochemistry, 73. 2365-2369. 2009.

[19] Guertler, P., Paul, V., Steinke, K., Wiedemann, S., Preißinger, W., Albrecht, C., Spiekers, H., Schwarz, FJ., Meyer, H.H.D. "Longterm feeding of genetically modified corn (MON810)-Fate of cry $1 \mathrm{~A} b$ DNA and recombinant protein during the metabolism of the dairy cow," Livestock Science, 131. 250-259. 2010.

[20] EUR, "European Union Reference Laboratory for GM food \& feed." 2013. [Online] Available: http://gmo-crl.jrc.ec.europa.eu

[21] Nap, J.P., Metz, P.L.J., Escaler, M., Conner, A.J., "The release of genetically modified crops into the environment," The Plant Journal, 33 (1). 1-18. Jan. 2003. 